\title{
Monte Carlo dosimetric study of the Flexisource Co-60 high dose rate source
}

\author{
Javier Vijande, Prof!.', Domingo Granero, PhD², Jose Perez-Calatayud, PhD ${ }^{45}$, Facundo Ballester, Prof! \\ 'Department of Atomic, Molecular and Nuclear Physics, University of Valencia, 2IFIC (CSIC-University of Valenica), Paterna (Valencia), Spain,

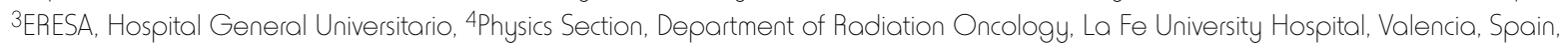 \\ 5Department of Radiotherapy, Clinica Benidorm, Valencia, Spain
}

\begin{abstract}
Purpose: Recently, a new HDR ${ }^{60}$ Co brachytherapy source, Flexisource Co-60, has been developed (Nucletron B.V. Veenendaal, The Netherlands). This study aims to obtain dosimetric data for this source for its use in clinical practice as required by AAPM and ESTRO.

Material and methods: Two Monte Carlo radiation transport codes were used: Penelope2008 and GEANT4. The source was centrally-positioned in a $100 \mathrm{~cm}$ radius water phantom. Absorbed dose and collisional kerma were obtained using $0.01 \mathrm{~cm}$ (close) and $0.1 \mathrm{~cm}$ (far) sized voxels to provide high-resolution dosimetry near (far from) the source. Dose rate distributions obtained with the two Monte Carlo codes were compared.

Results and Discussion: Simulations performed with those two radiation transport codes showed an agreement typically within $0.2 \%$ for $r>0.8 \mathrm{~cm}$ and up to $2 \%$ closer to the source. Detailed results of dose distributions are being made available.

Conclusions: Dosimetric data are provided for the new Flexisource Co-60 source. These data are meant to be used in treatment planning systems in clinical practice.

Key words: Flexisource Co-60, brachytherapy, TG-43, Penelope2008, GEANT4.

\section{Purpose}

According to the American Association of Physicist in Medicine (AAPM) and the European Society for Radiotherapy and Oncology (ESTRO) recommendations [1], in order to fulfil the dosimetric prerequisites, all sources to be used in clinical practice have to have a set of dosimetric parameters available based on the Radiation Therapy Committee Task Group No. 43 (TG-43) formalism [2,3]. The AAPM High Energy Brachytherapy Source Dosimetry Working Group (HEBD-WG) recommends [1] that this dataset must be based upon at least one experimental study and at least one Monte Carlo (MC) study of the model's source dosimetric parameters. For conventionally encapsulated sources similar in design to existing or previously existing ones, a single dosimetric study published in a peer-reviewed journal is sufficient. The high dose rate (HDR) ${ }^{60} \mathrm{Co}$ sources fall in this category. MC or experimental dosimetry (or both) methods may be used. These studies must be performed by investigators that are independent from the manufacturer and published in a peer-reviewed journal prior to the use of these isotopes in clinical practice.

The HEBD-WG is also concerned about the dosimetry in the near-source region where the influence of $\beta$ electrons and the lack of electronic equilibrium are frequently neglected [4-6]. Commercial treatment planning systems (TPS) allow direct introduction of tabulated dose rates from the literature using the TG- 43 formalism. These TG- 43 data are usually derived from $\mathrm{MC}$ radiation transport simulations, estimating absorbed dose by collisional kerma. Consequently, these data are provided at distances from the source capsule large enough to ensure that the equivalence of collisional kerma and dose is applicable. TPS extrapolate data outside the available TG-43 data range. In case of HDR ${ }^{60} \mathrm{Co}$ sources, kerma to dose differences are significant and source model specific [4]. Kerma extrapolation at short distances would not be necessary if TG-43 data were available with adequate range and spatial resolution that include source electron contributions to absorbed dose and account for electron disequilibrium.

High dose rate (HDR) brachytherapy ${ }^{60} \mathrm{Co}$ sources have been considered for use in clinical practice as an alternative for ${ }^{192}$ Ir HDR sources [7]. A comparison between the radiological properties of cobalt and iridium HDR sources have been performed in reference [8]. Additionally to cost and logistics improvements due to the cobalt longer half life, clinical examples for intracavitary and interstitial applications show practically identical dose distributions. 
The main goal of this work is to present the TG-43 data and the 2D dose rate table in cylindrical coordinates for treatment planning and quality assurance purposes (QA) for the new Flexisource Co-60 HDR source model used by the Flexitron remote HDR afterloader (Nucletron B.V., Veenendaal, The Netherlands) in a consistent way that is valid at short and long distances. Such a source has not been studied and published previously.

\section{Material and methods}

The design and materials of the Flexisource Co-60 HDR source was provided by the manufacturer. The source design and dimensions are shown schematically in Fig. 1. It is composed of a central cylindrical active core made of metallic ${ }^{60} \mathrm{Co}$ with a density of $8.9 \mathrm{~g} / \mathrm{cm}^{3}, 3.5 \mathrm{~mm}$ in length and $0.5 \mathrm{~mm}$ in diameter. The active core is covered by a cylindrical 316L stainless steel $(67 \% \mathrm{Fe}, 11 \% \mathrm{Ni}, 18 \% \mathrm{Cr}$, $2 \% \mathrm{Si}, 2 \% \mathrm{Mn}$ ) layer of $0.9 \mathrm{~mm}$ of external diameter and a density of $8 \mathrm{~g} / \mathrm{cm}^{3}$. For this study we considered $2 \mathrm{~mm}$ $316 \mathrm{~L}$ steel cable with an effective density of $4.81 \mathrm{~g} / \mathrm{cm}^{3}$ (measured from the inner clamp). The interstitial areas between the active element and the cover were considered to be filled with standard dry air.

MC methods for radiation transport simulations were used to study the dose rate distribution around the source. Different MC codes presented different physics models, different cross sections data, and dissimilar tracking methods in the transport of electrons. As dose at short distances from the source was required, where electronic disequilibrium conditions may be dosimetrically important, two different MC codes were used. These MC codes were Penelope2008 [9] and GEANT4 (version 9.3) [10], which have been successfully used for dosimetric studies in the field of the brachytherapy [4,11-15].

GEANT4 and Penelope2008 photon and electron crosssections are based on the EPDL97 and EEDL97 cross sections libraries, respectively [16, 17]. However, Penelope2008 also considers the impulse approximation that accounts for Doppler broadening and binding effects [9]. Consequently, photoelectric effect, pair production and Rayleigh cross-sections used by both codes were the same, while Compton cross-sections in Penelope2008 differed from those of GEANT4. Possible influence on the dosimetric results by using Penelope2008 with the Compton cross-sections of the EPDL97 library has been discussed elsewhere [14] and found to be negligible. The photon spectrum was taken from the NuDat database [18] as suggested in Ref. [19]. The number of photons $N_{\gamma}$ generated in each simulation was as follows: Penelope2008 $\left(N_{\gamma}=5 \times 10^{9}\right)$, GEANT4 $\left(N_{\gamma}=1 \times 10^{9}\right.$ to obtain water kerma and $N_{\gamma}=4 \times 10^{9}$ to calculate absorbed dose). The electron spectrum including $\beta$ decay, internal conversion electrons (IC) and Auger electrons was not considered in the simulation since its effect over the total dose was known to be less than $1 \%$ at distances greater than $0.1 \mathrm{~cm}$ from the source surface [4]. In each disintegration, 2.0001 photons / $(\mathrm{Bq} \mathrm{s})$ were generated on average. However, due to the $10 \mathrm{keV}$ cut-off used, the photon intensity was reduced to 1.9985 photons/(Bq s).

Dose to water contribution was calculated in Penelope2008 using the tally provided within the penEasy package [20], whereas in GEANT4 it was evaluated using a homemade routine with the function GetTotalEnergyDeposit of the GEANT4 toolkit. To estimate the collisional kerma, homemade routines using the linear track-length estimator [21] were developed for Penelope2008 and GEANT4. Dose and collisional kerma rate distributions were used to derive the final dosimetric parameters as a function of $r$ at every polar angle sampled.

The source was located at the geometric center of a spherical liquid water phantom with $100 \mathrm{~cm}$ radius, to estimate dose to water and simulate unbounded phantom conditions for $r<20 \mathrm{~cm}$ [22]. Water composition and mass density were those recommended by the AAPM [3]. Due to the high energy of the ${ }^{60} \mathrm{Co}$, the photon spectrum electronic equilibrium is not reached up to a distance of approximately $0.75 \mathrm{~cm}$ from the surface of the source [4]. Thus, the dose for small distances cannot be approximated by collisional kerma as is usually done for ${ }^{192}$ Ir or ${ }^{137}$ Cs sources. Differences between collisional kerma and dose at $r=0.75 \mathrm{~cm}$ are less than $0.5 \%$ and negligible at $r=1 \mathrm{~cm}$ [4]. Since the evaluation of collisional kerma was more efficient (reduced statistical uncertainty and improved numerical performance) we have considered absorbed dose to water for distances smaller than $0.75 \mathrm{~cm}$ and collisional kerma from $0.75 \mathrm{~cm}$ up to $20 \mathrm{~cm}$. In order to provide adequate spatial resolution, the cells were $0.01 \mathrm{~cm}$ in thickness for $r<2 \mathrm{~cm}$ from the source and a factor of 10 thicker for $2 \mathrm{~cm}<r<20 \mathrm{~cm}$, respe-

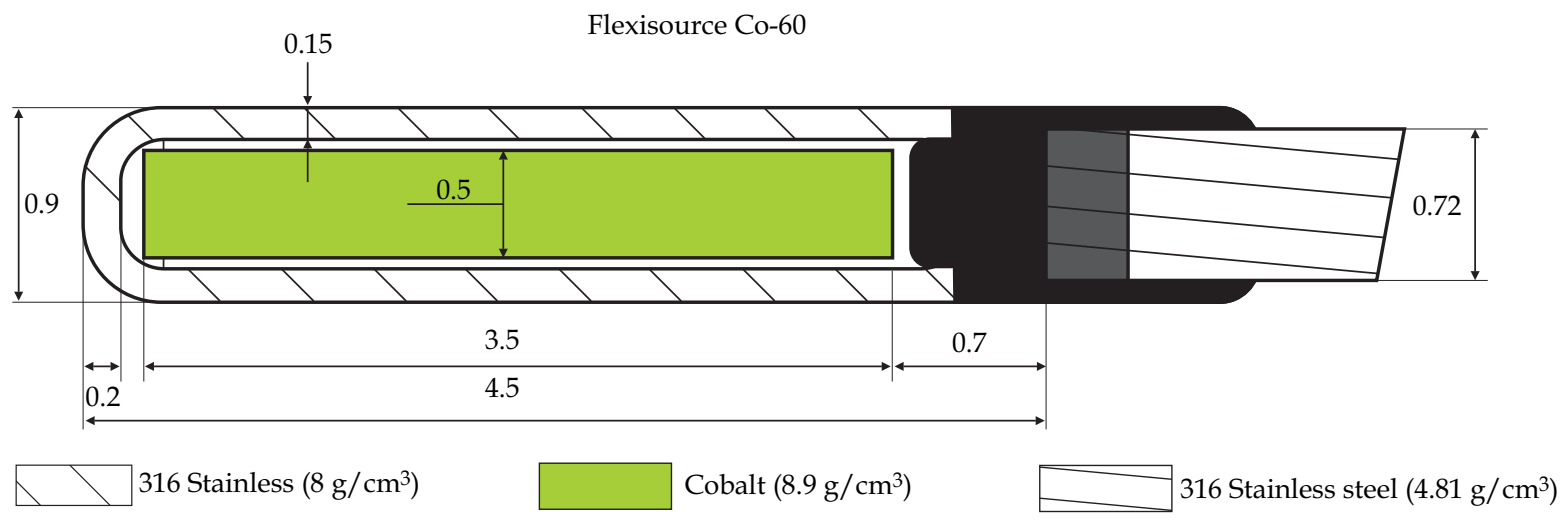

Fig. 1. Schematic design and dimensions of the model Flexisource Co-60 HDR source. Dimensions are given in mm 
Table 1. Comparison of dose rate constant values calculated for similar ${ }^{60} \mathrm{Co}$ HDR sources

\begin{tabular}{lccc} 
& $\begin{array}{c}L \\
(\mathrm{~cm})\end{array}$ & $\boldsymbol{\Lambda}(\mathrm{cGy} \mathrm{h}-1 \mathrm{U}-1)$ & $\begin{array}{c}\Lambda / G\left(r=1 \mathrm{~cm}, \boldsymbol{\theta}=90^{\circ}\right) \\
\left(\mathrm{cGy} \mathrm{cm} \mathrm{h}^{-1} \mathrm{U}-1\right)\end{array}$ \\
\hline Flexisource Co-60 (This work) & 0.35 & $1.085 \pm 0.003$ & 1.096 \\
\hline BEBIG Multisource GK60M21 [10] & 0.35 & $1.084 \pm 0.005$ & 1.095 \\
\hline BEBIG Multisource Co0.A86 [12] & 0.35 & $1.090 \pm 0.010$ & 1.098 \\
\hline Ralstron Type 2 [8] & 0.20 & $1.101 \pm 0.005$ & 1.105 \\
\hline GZP6 source (Ch. 6) [13] & 0.35 & $1.086 \pm 0.005$ & 1.097 \\
\hline GZP6 source (Ch. 3/4) [13] & 0.35 & $1.087 \pm 0.005$ & 1.098
\end{tabular}

Table 2. Radial dose function calculated for the Flexisource Co-60 HDR source

\begin{tabular}{|c|c|}
\hline$r(\mathrm{~cm})$ & $g_{L}(r)$ \\
\hline 0.1 & 0.837 \\
\hline 0.15 & 0.971 \\
\hline 0.2 & 1.045 \\
\hline 0.22 & 1.065 \\
\hline 0.25 & 1.079 \\
\hline 0.27 & 1.081 \\
\hline 0.3 & 1.080 \\
\hline 0.4 & 1.054 \\
\hline 0.5 & 1.031 \\
\hline 0.6 & 1.021 \\
\hline 0.7 & 1.012 \\
\hline 0.8 & 1.004 \\
\hline 0.9 & 1.002 \\
\hline 1 & 1.000 \\
\hline 1.5 & 0.992 \\
\hline 2 & 0.984 \\
\hline 2.5 & 0.976 \\
\hline 3 & 0.967 \\
\hline 3.5 & 0.960 \\
\hline 4 & 0.951 \\
\hline 4.5 & 0.944 \\
\hline 5 & 0.935 \\
\hline 6 & 0.919 \\
\hline 7 & 0.902 \\
\hline 8 & 0.885 \\
\hline 9 & 0.868 \\
\hline 10 & 0.850 \\
\hline 11 & 0.832 \\
\hline 12 & 0.814 \\
\hline 14 & 0.777 \\
\hline 16 & 0.739 \\
\hline 18 & 0.701 \\
\hline 20 & 0.663 \\
\hline
\end{tabular}

ctively. Collisional kerma and absorbed dose were obtained simultaneously in cylindrical $(y, z)$ and spherical $(r, \theta)$ coordinates. Angular sampling was taken every $2^{\circ}$.

Additional simulations were performed to obtain $S_{K}$ with the source surrounded by vacuum, except for a small cylindrical air cell of $0.1 \mathrm{~cm}$ in diameter and $0.1 \mathrm{~cm}$ in height at $r=10 \mathrm{~cm}$, as recommended by AAPM [3]. Mass-energy absorption coefficients in water and air were consistently derived for each code and used to calculate the collisional kerma.

\section{Results}

The dose rate distribution $\dot{D}(r, \theta)$ for the Flexisource Co-60 HDR source model constructed as described in Sect. Material and Methods was used to derive the TG-43 dosimetry parameters with $L=0.35 \mathrm{~cm}$. Using Penelope2008 and GEANT4, an average of $\Lambda=1.085 \pm 0.003 \mathrm{cGy} /(\mathrm{h} \mathrm{U})$ (with $k=1$ ) was obtained. Uncertainties are Type A only. These are similar to the consensus values published for other ${ }^{60}$ Co sources, see Table 1 . In Table 2 and $3 g_{L}(r)$ and $F(r, \theta)$ are provided in $0.1 \mathrm{~cm}$ steps (or smaller) up to $1.0 \mathrm{~cm}$ from the source (to reproduce the dose distribution accurately at close distances) and in $0.5 \mathrm{~cm}$ and $1 \mathrm{~cm}$ steps up to $20 \mathrm{~cm}$. Both functions were obtained as average results from Penelope2008 and GEANT4 codes. $F(r, \theta)$ was provided for all radial distances in $2^{\circ}$ increments. An along-away table for QA purposes is also provided in Table 4.

Differences in using $D(r, \theta)$ Penelope2008 and GEANT4 were within the statistical uncertainties (type A). These uncertainties were larger at $r<1 \mathrm{~cm}$ where absorbed dose were scored (between $0.5 \%$ at $r=0.2 \mathrm{~cm}$ and $1 \%$ at $r=0.8 \mathrm{~cm}$ ) and lower at larger distances (below $0.1 \%$ ) where the collisional kerma was used.

\section{Discussions}

In this study, we have compared our results with those obtained for other ${ }^{60} \mathrm{Co}$ sources discussed in the literature. Papagiannis et al. [23] used MC to obtain dose rate in water $(30 \mathrm{~cm}$ in diameter water phantom) of the Ralstron Type-1, Type- 2 and Type- 3 source models manufacured by Shimazdu Corporation (Japan) and used in the Ralstron remote afterloader. Their configuration consists of two active pellets (cylinders $1 \mathrm{~mm} \times 1 \mathrm{~mm}$ ) either in contact (Type-2), $9 \mathrm{~mm}$ (Type-1) or $11 \mathrm{~mm}$ apart (Type-3). All three models have a $3 \mathrm{~mm}$ external diameter. Kerma-dose approxima- 
Table 3. Anisotropy function calculated for the Flexisource Co-60 HDR source

\begin{tabular}{|c|c|c|c|c|c|c|c|c|c|c|c|c|c|c|c|c|c|}
\hline \multicolumn{18}{|c|}{$\mathrm{r} / \mathrm{cm}$} \\
\hline$\theta^{\circ}$ & 0.1 & & 2 & & & & 3 & & 0.5 & & & & & & 5 & & .5 \\
\hline 0 & $\mathrm{NaN}$ & $\mathrm{NaN}$ & $\mathrm{NaN}$ & $\mathrm{NaN}$ & 0.653 & .696 & .765 & .920 & & 955 & & .945 & 0.948 & 950 & 0.948 & 952 & 0.952 \\
\hline 2 & $\mathrm{NaN}$ & $\mathrm{NaN}$ & & $\mathrm{NaN}$ & & & & & & & & & & & & & \\
\hline 4 & $\mathrm{NaN}$ & & & & & & & & & & & & & & & & \\
\hline 6 & $\mathrm{NaN}$ & $\mathrm{N}$ & & & & & & & & & & & & & & & \\
\hline 8 & $\mathrm{NaN}$ & $\mathrm{NaN}$ & $\mathrm{aN}$ & $\mathrm{NaN}$ & 667 & .711 & 775 & & 977 & & & & & & 963 & 963 & \\
\hline 10 & $\mathrm{NaN}$ & NaN & $\mathrm{NaN}$ & $\mathrm{NaN}$ & 0.675 & 0.717 & 0.781 & 0.935 & 0.977 & 976 & 975 & 0.969 & 969 & 969 & .967 & 967 & 0.967 \\
\hline 12 & $\mathrm{NaN}$ & $\mathrm{NaN}$ & $\mathrm{NaN}$ & $\mathrm{NaN}$ & 0.685 & 0.727 & 0.790 & 0.936 & 0.979 & 978 & 983 & 0.972 & 0.971 & 972 & 0.972 & .971 & 0.972 \\
\hline 14 & Nave & $\mathrm{NaN}$ & & 610 & 0.690 & & 800 & & & & & & & & & & \\
\hline 16 & $\mathrm{NaN}$ & $\mathrm{NaN}$ & & & & & & & & & & & & & & & \\
\hline 18 & $\mathrm{NaN}$ & & & 665 & .721 & & & & 0.989 & & & 0.982 & & & 0.981 & & 0.981 \\
\hline 20 & $\mathrm{NaN}$ & $\mathrm{NaN}$ & 75 & 695 & .743 & .780 & 0.834 & & 0.992 & & & 0.984 & & & 0.985 & 84 & 0.984 \\
\hline 22 & $\mathrm{NaN}$ & 0.703 & 706 & 723 & 0.764 & 0.797 & 0.847 & & 0.993 & & & 0.986 & & 986 & 985 & 985 & 0.986 \\
\hline 24 & $\mathrm{NaN}$ & 0724 & 732 & .748 & 0.785 & 0.815 & 0.861 & 0.964 & 0.994 & 0.991 & & 0.987 & & 988 & 0989 & 987 & 0.988 \\
\hline 26 & $\mathrm{NaN}$ & 0.742 & 757 & .772 & 0.806 & & 0.874 & 0.970 & 0.997 & 0.994 & & 0.989 & & & & & 0.989 \\
\hline 28 & $\mathrm{NaN}$ & 764 & & 793 & 0.824 & 0.848 & 0.886 & & 0.997 & & & 001 & & & م0000 & & 0.990 \\
\hline 30 & $\mathrm{NaN}$ & & & & & & & & 0.996 & & & & & & & & 0.992 \\
\hline 32 & $\mathrm{NaN}$ & 195 & & 832 & .859 & 0.879 & .910 & & 0.998 & & 0.999 & 0.993 & 0.992 & & 0.992 & 92 & 0.992 \\
\hline 34 & $\mathrm{NaN}$ & 813 & 335 & 849 & 0.874 & 0.893 & 0.921 & & 1.000 & 994 & 998 & 0.993 & 0.993 & 93 & 0.994 & 993 & 0.993 \\
\hline 36 & $\mathrm{NaN}$ & 830 & 852 & .864 & 0.888 & 0.905 & 0.930 & 0.990 & 1001 & 0.997 & 0.997 & 0.995 & 0.995 & .995 & 0.994 & 994 & 0.994 \\
\hline 38 & 0.824 & 843 & 866 & 0.877 & 0.898 & 0.914 & 0.937 & 0.991 & 1002 & 0.997 & 0.998 & 0.995 & 0.995 & 996 & 0995 & 995 & 0.995 \\
\hline 40 & .839 & 857 & 881 & 892 & 0.910 & 0.924 & 0.945 & 0.995 & 1.005 & & & 0.995 & 0.995 & 006 & 095 & 995 & 0.995 \\
\hline 42 & & & & & & & & & & & & & & & & & \\
\hline 44 & $.8 / 5$ & 882 & & 913 & 0.929 & .940 & 0.959 & & 1.005 & & 000 & 0.996 & & & 0.997 & 996 & 0.996 \\
\hline 46 & 892 & 895 & & 924 & .939 & .949 & .965 & & 1.002 & & & 0.997 & & & 0.997 & 96 & 0. \\
\hline 48 & 905 & 905 & 923 & 932 & .946 & 0.955 & 0.968 & & 1.003 & & 998 & 0.997 & 98 & & 0.998 & 997 & 0.998 \\
\hline 50 & 915 & 916 & 932 & 0.939 & 0.952 & 0.962 & 0.975 & & 1003 & & 1.004 & 0.998 & 0.998 & & 0989 & & 0.997 \\
\hline 52 & 924 & 924 & 938 & 0.946 & 0.958 & 0.966 & 0.978 & 0.999 & 1004 & 0.999 & .005 & 0.998 & 0.998 & .998 & 0998 & 998 & 0.998 \\
\hline 54 & 0.933 & 932 & 946 & 0.953 & 0.965 & 0.973 & 0.983 & & 1.001 & & & 0.998 & & & 0 & & 0.998 \\
\hline 56 & תנת. & 942 & & .959 & 0.968 & 0.974 & 0.984 & & 1.004 & & & 0.998 & & & & & 0.998 \\
\hline 58 & 943 & 948 & & 966 & & & & & 1.005 & & & & & & & & 0.998 \\
\hline 60 & 953 & 955 & 964 & 969 & 0.978 & 0.983 & 0.990 & 1.004 & 1.002 & 000 & 003 & 0.999 & 0.999 & 99 & 0.999 & 999 & 0.999 \\
\hline 62 & 960 & 959 & 969 & .973 & 0.980 & 0.984 & 0.991 & 1.0 & 1.002 & 1.001 & 1.003 & 0.998 & 1.000 & .000 & 0.999 & 999 & 0.999 \\
\hline 64 & .964 & 966 & 973 & .976 & 0.981 & 0.985 & 0.991 & 1.003 & 1002 & 1.001 & 1.005 & 0.999 & 1.000 & مอم & 1.000 & 999 & 0.999 \\
\hline 66 & 972 & 973 & & 980 & 0.985 & 0.989 & 0.994 & 1.001 & 1002 & & & ק & 0.999 & 0.999 & ק & & 0.999 \\
\hline 68 & .979 & 976 & 981 & .984 & 0.988 & 0.991 & & & 1.00 & & & (19) & & & 1.000 & & 0.999 \\
\hline 70 & 0.983 & 981 & 984 & .986 & 0.991 & 0.994 & 0.997 & & 1.001 & & & 0.999 & & & 1.000 & & 0.999 \\
\hline 72 & 0.987 & 983 & & 989 & 0.992 & 0.995 & 0.998 & & 1.002 & & & 1.0 & & & 1.000 & & 1.000 \\
\hline 74 & 993 & 985 & 990 & 992 & 0.995 & 0.997 & 1.000 & 1.0 & 1.001 & & 02 & 1.000 & 1.0 & 0 & 0.999 & 999 & 0.999 \\
\hline 76 & 0.995 & 991 & & .995 & 0.99 & & & & 1.001 & & & 1.000 & & 1 & 1.000 & 999 & 0.999 \\
\hline 78 & 0.998 & 0.990 & 993 & 0.995 & 0.997 & 0.998 & 1.000 & 1.001 & 0.999 & 1.003 & 1.001 & 1.000 & 1.000 & 1.000 & 1.000 & 0.999 & 1.000 \\
\hline 80 & 1.001 & 0.994 & 996 & 0.997 & 0.998 & 0.999 & 1.000 & 1.001 & 0.999 & & 1002 & 1.000 & 1.000 & 1.000 & 0.999 & 0.999 & 0.999 \\
\hline 82 & 1.005 & 0.995 & 996 & 0.997 & 0.998 & 0.998 & 1.000 & 1.000 & 0.999 & 1.000 & 1.003 & 1.000 & 1.000 & 1.000 & 1.000 & 1.000 & 1.000 \\
\hline 84 & 1.004 & 995 & 996 & 0.997 & 0.999 & 0.999 & 0.999 & 1.000 & 1.000 & 1.001 & 1.004 & 1.000 & 1.000 & 1.000 & 1.000 & 0.999 & 0.999 \\
\hline 86 & 1.006 & 0.997 & .998 & 998 & 0.998 & 0.998 & 0.999 & 0.999 & 0.999 & 1.003 & 1.003 & 1.000 & 1.000 & 1.000 & 1.000 & 0.999 & 0.999 \\
\hline 88 & 1.008 & 0.999 & 000 & 999 & 0.998 & 0.998 & 0.998 & 0.998 & 1.002 & 1.002 & 1005 & 1.000 & 1.001 & 1.001 & 1.000 & 000 & 1000 \\
\hline
\end{tabular}


Table 3. Cont.

\begin{tabular}{|c|c|c|c|c|c|c|c|c|c|c|c|c|c|c|c|c|}
\hline \multicolumn{17}{|c|}{$\mathrm{r} / \mathrm{cm}$} \\
\hline$\theta^{\circ}$ & & 5 & 4 & .5 & 5 & 6 & 7 & 8 & 9 & 10 & 11 & 12 & 14 & 16. & 18 & 20 \\
\hline 0 & 0.953 & 0.952 & 0.954 & 0.954 & 0.955 & 0.956 & 0.957 & 0.960 & 0.961 & 0.961 & 0.963 & 0.963 & 0.966 & 0.967 & 0.969 & 0.986 \\
\hline 2 & 956 & 956 & & 957 & .958 & .959 & 960 & 0.962 & 962 & 0.963 & 0.965 & 0.965 & 0.967 & 0.968 & 0.970 & 0.97 \\
\hline 4 & 960 & 960 & & 0.960 & 961 & & 0.963 & 0.964 & & & 0.966 & 0.967 & & & 0.971 & \\
\hline 6 & 960 & 960 & 61 & 961 & 962 & 962 & 963 & 0.963 & 964 & 965 & 0.966 & 967 & 0.967 & 969 & 0.970 & $0.9 / 1$ \\
\hline 8 & .964 & 0.964 & 965 & 0.965 & 0.966 & 0.966 & 0.967 & 0.968 & 0.968 & 0.969 & 0.969 & 0.970 & 0.971 & 0.972 & 0.973 & 0.973 \\
\hline 10 & 0.968 & 0.968 & 0.968 & 0.969 & 0.969 & 0.969 & 0.970 & 0.970 & 0.971 & 0.972 & 0.972 & 0.973 & 0.973 & 0.974 & 0.975 & 0.976 \\
\hline 12 & .972 & 0.972 & .973 & 0.973 & 0.973 & 0.974 & 0.975 & 0.975 & 0.975 & 0.976 & 0.976 & 0.977 & 0.977 & 0.978 & 0.979 & 0.979 \\
\hline 14 & 976 & 0.976 & 976 & 0.976 & 0.976 & 0.977 & 0.977 & 0.978 & 0.978 & 0.978 & 0.979 & 0.979 & 0.980 & 0.980 & 0.981 & 0.981 \\
\hline 16 & 978 & 0.978 & 978 & 0.979 & 0.979 & 0.979 & 0.980 & 0.980 & 0.980 & 0.980 & 0.981 & 0.981 & 0.981 & 0.982 & 0.982 & 0.98 \\
\hline 18 & 981 & 0.981 & 982 & 0.982 & 0.982 & 0.983 & 0.983 & 0.983 & 0.983 & 0.983 & 0.984 & 0.984 & 0.984 & 0.985 & 0.985 & 0.985 \\
\hline 20 & 984 & 0.984 & 0.985 & 0.985 & 0.985 & 0.985 & 0.986 & 0.986 & 0.986 & 0.986 & 0.986 & 0.987 & 0.986 & 0.987 & 0.987 & 0.98 \\
\hline 22 & 0.986 & 0.986 & 0.986 & 0.986 & 0.986 & 0.986 & 0.987 & 0.987 & 0.987 & 0.987 & 0.987 & 0.987 & 0.987 & 0.988 & 0.988 & 0.988 \\
\hline 24 & 0.988 & 0.988 & 0.988 & 0.988 & 0.989 & 0.989 & 0.989 & 0.989 & 0.989 & 0.989 & 0.989 & 0.989 & 0.989 & 0.989 & 0.990 & 0.99 \\
\hline 26 & 0.989 & 0.989 & 0.989 & 0.989 & 0.989 & 0.990 & 0.990 & 0.990 & 0.990 & 0.990 & 0.990 & 0.990 & 0.990 & 0.991 & 0.991 & 099 \\
\hline 28 & 990 & 0.990 & .991 & 0.990 & 0.991 & 0.991 & 0.991 & 0.991 & 0.991 & 0.991 & 0.991 & 0.991 & 0.991 & 0.991 & 0.992 & 099 \\
\hline 30 & 992 & 0.992 & 992 & 0.992 & 0.992 & 0.992 & 0.992 & 0.992 & 0.992 & 0.992 & 0.992 & 0.992 & 0.993 & 0.993 & 0.993 & 0.96 \\
\hline 32 & 992 & 0.992 & 992 & 0.992 & 0.992 & 0.993 & 0.993 & 0.993 & 0.992 & 0.993 & 0.993 & 0.993 & 0.993 & 0.993 & 0.993 & 0.99 \\
\hline 34 & .994 & 0.994 & 0.994 & 0.994 & 0.994 & 0.994 & 0.994 & 0.994 & 0.994 & 0.994 & 0.994 & 0.994 & 0.994 & 0.994 & 0.995 & 0.99 \\
\hline 36 & 0.994 & 0.994 & 0.995 & 0.994 & 0.995 & 0.995 & 0.995 & 0.995 & 0.995 & 0.995 & 0.995 & 0.995 & 0.995 & 0.995 & 0.995 & 0.99 \\
\hline 38 & 0.995 & 0.995 & 0.995 & 0.995 & 0.995 & 0.995 & 0.995 & 0.995 & 0.995 & 0.995 & 0.995 & 0.995 & 0.995 & 0.995 & 0.995 & 0.99 \\
\hline 40 & 0.995 & 0.995 & 0.995 & 0.995 & 0.995 & 0.995 & 0.995 & 0.995 & 0995 & 0.995 & 0995 & 0.995 & 0.995 & 099 & 0.995 & 099 \\
\hline 42 & & 0.996 & 0.996 & 0.996 & 0.996 & 0.996 & 0.996 & 0.996 & 0.990 & 0.996 & 0.00 & 0.996 & 0.996 & 0.50 & 0.996 & (.) \\
\hline 44 & 996 & 0.996 & 997 & 0.997 & 0.997 & 0.997 & 0.997 & 0.997 & 0.997 & 0.997 & 0.997 & 0.997 & 0.997 & 0.997 & 0.997 & 0.99 \\
\hline 46 & 996 & .996 & 997 & 0.997 & 0.996 & 0.997 & 0.997 & 0.997 & 0.996 & 0.996 & 0.996 & 0.996 & 0.996 & 0.996 & 0.996 & 0.99 \\
\hline 48 & .998 & 0.998 & 0.998 & 0.998 & 0.998 & 0.998 & 0.998 & 0.998 & 0.998 & 0.998 & 0.998 & 0.998 & 0.998 & 0.998 & 0.998 & 0.99 \\
\hline 50 & 0.998 & 0.998 & 0.998 & 0.998 & 0.998 & 0.998 & 0.998 & 0.998 & 0.998 & 0.998 & 0.998 & 0.998 & 0.998 & 0.998 & 0.998 & 0.99 \\
\hline 52 & .999 & 0.999 & 0.999 & 0.999 & 0.999 & 0.999 & 0.999 & 0.999 & 0 & 0.999 & 0.999 & 0.999 & 0.999 & 0.999 & 0.999 & 0.99 \\
\hline 54 & 998 & 0.998 & 0.998 & 0.998 & 0.998 & 0.998 & 0.998 & 0.998 & 0.998 & 0.998 & 0.999 & 0.998 & 0.998 & 0.998 & 0.999 & 09 \\
\hline 56 & 998 & 0.998 & 999 & 0.999 & 0.999 & 0.999 & 0.999 & 0.998 & 0.998 & 0.999 & 0.999 & 0.999 & 0.999 & 0.999 & 0.999 & 0.9 \\
\hline 58 & 999 & 998 & 999 & 0.999 & 0.999 & 0.999 & 0.999 & 0.999 & 0.999 & 0.999 & 0.999 & 0.999 & 0.999 & 0.999 & 0.999 & 0.9 \\
\hline 60 & 999 & 0.999 & 0.999 & 0.999 & 0.999 & 0.999 & 0.999 & 0.999 & 0.999 & 0.999 & 0.999 & 0.999 & 0.999 & 0.999 & 0.999 & 0.99 \\
\hline 62 & 0.999 & 0.999 & 0.999 & 0.999 & 0.999 & 0.999 & 0.999 & 0.999 & 0.999 & 0.999 & 0.999 & 0.999 & 0.999 & 0.999 & 0.999 & 0.99 \\
\hline 64 & 0.999 & 0.999 & 1.000 & 0.999 & 0.999 & 1.000 & 1.000 & 0.999 & 0.999 & 0.999 & 1.000 & 0.999 & 1.000 & 1.000 & 1.000 & 0.99 \\
\hline 66 & 0.999 & 0.999 & 0.999 & 0.999 & 0.999 & 0.999 & 0.999 & 0.999 & 0.999 & 0.999 & 0.999 & 0.999 & 0.999 & 0.999 & 0.999 & 0.9 \\
\hline 68 & 0.999 & 0.999 & 0.999 & 0.999 & 0.999 & 0.999 & 0.999 & 0.999 & 0.999 & 0.999 & 0.999 & 0.999 & 0.999 & 0.999 & 1.000 & 0.9 \\
\hline 70 & 0.999 & 0.999 & 1.000 & 0.999 & 1.000 & 1.000 & 1.000 & 1.000 & 0.999 & 1.000 & 1.000 & 1.000 & 1.000 & 1.000 & 1.000 & 1.0 \\
\hline 72 & 1.000 & 1.000 & Do0 & 1.000 & 1.000 & .000 & 1.000 & 1.000 & 1.000 & 1.000 & 1.000 & 1.000 & 1.000 & 1.000 & 1.000 & 1.0 \\
\hline 74 & 0.999 & 0.999 & 0.999 & 999 & 0.999 & 0.999 & 1.000 & 0.999 & 0.999 & 0.999 & 0.999 & 0.999 & 0.999 & 0.999 & 1.000 & 0.9 \\
\hline 76 & 0.999 & 0.999 & 0.999 & 0.999 & 0.999 & 0.999 & 0.999 & 0.999 & 0.999 & 0.999 & 0.999 & 0.999 & 0.999 & 0.999 & 0.999 & 0.99 \\
\hline 78 & 1.000 & 1.000 & 1.000 & 1.000 & 1.000 & 1.000 & 1.000 & 1.000 & 1.000 & 1.000 & 1.000 & 1.000 & 1.000 & 0.999 & 1.000 & 100 \\
\hline 80 & 0.999 & 0.999 & 0.999 & 0.999 & 0.999 & 0.999 & 1.000 & 0.999 & 0.999 & 0.999 & 0.999 & 0.999 & 1.000 & 0.999 & 0.999 & 0.99 \\
\hline 82 & 1.000 & 1.000 & 1.000 & 1.000 & 1.000 & 1.000 & 1.000 & 1.000 & 1.000 & 1.000 & 1.000 & 1.000 & 1.000 & 1.000 & 1.000 & 1.00 \\
\hline 84 & 1.000 & 1.000 & 1.000 & 1.000 & 1.000 & 1.000 & 1.000 & 1.000 & 0.999 & 1.000 & 0.999 & 0.999 & 1.000 & 1.000 & 1.000 & 1.000 \\
\hline 86 & 1.000 & 1.000 & 1.000 & 1.000 & 1.000 & 1.000 & 1.000 & 1.000 & 1.000 & 1.000 & 1.000 & 1.000 & 1.000 & 1.000 & 1.000 & 1.00 \\
\hline 88 & 1.000 & 1.000 & 1.000 & 1.000 & 1.000 & 1.000 & 1.000 & 1.000 & 1.000 & 1.000 & 1.000 & 1.000 & 1.000 & 1.000 & 1.000 & 1.00 \\
\hline
\end{tabular}


Table 3. Cont

\begin{tabular}{|c|c|c|c|c|c|c|c|c|c|c|c|c|c|c|c|c|c|}
\hline \multicolumn{18}{|c|}{ 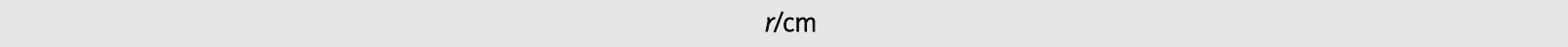 } \\
\hline$\theta^{\circ}$ & & 15 & & .22 & & .27 & 0.3 & 0.4 & 5 & 0.6 & 0.7 & 0.8 & .9 & 1 & .5 & 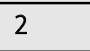 & 2.5 \\
\hline 90 & 1.000 & 000 & 000 & 000 & 1.000 & 1.000 & .000 & 000 & & & & 1.000 & 1.000 & 0 & 1.000 & .000 & 1.000 \\
\hline 92 & 1.005 & & & & & & & & & & & & & & & Do & \\
\hline 94 & & & & & & & & & & & & & & & & 00 & \\
\hline 96 & .006 & & & & 1.000 & & & & & & & & & & & & \\
\hline 98 & & & & & & & & & & & & & & & & & \\
\hline 100 & 997 & & & & & & & & & & & & & & & 999 & 999 \\
\hline 102 & 7 & 1 & & & & & & & & & & & & & & 00 & \\
\hline 104 & 994 & .990 & 993 & 0.994 & 0.996 & 0.997 & 000 & 2 & 001 & & & & 000 & & & 999 & 1000 \\
\hline 106 & 992 & 987 & 992 & 993 & 0.996 & 0.997 & & & & & & & & & & .000 & \\
\hline 108 & 988 & & & 992 & 0.995 & .996 & & & & & & & & & & & 1000 \\
\hline 110 & 986 & & & 990 & 0.993 & & & & & & & & & & & & \\
\hline 112 & 977 & & & .987 & .991 & & & & & & & & & & & 999 & \\
\hline 114 & 976 & 972 & 978 & 981 & 0.987 & & & & & & & & & & & 999 & \\
\hline 116 & 969 & 967 & 978 & 0.982 & 0.988 & 0.991 & 996 & & & & & & & & & 999 & c \\
\hline 118 & 964 & 963 & 973 & 0.977 & 0.983 & 0.988 & & & & & & & & & & & \\
\hline 120 & 953 & .957 & 970 & 0.975 & 0.981 & 0.986 & & & & & & & & & & & \\
\hline 122 & 943 & 949 & & .971 & 0.980 & & & & & & & & & & & & \\
\hline & 938 & & & 900 & 0.976 & & & & & & & & & & & & \\
\hline 126 & 934 & 936 & & 961 & 0.972 & & & & & & & & & & & 998 & \\
\hline 128 & 925 & 928 & 945 & 953 & 0.965 & & & & & & & & & & & 98 & \\
\hline 130 & 915 & 0.918 & 939 & 0.947 & 0.961 & 0.971 & & & & & & & & & & 998 & \\
\hline 132 & 0.901 & 0.910 & 933 & 0.943 & 0.957 & 0.967 & & 1.00 & 004 & & & & & & & .998 & 0.998 \\
\hline 134 & 890 & 0.900 & 924 & 0.935 & 0.952 & 0.963 & & & & & & & & & & & \\
\hline 136 & & & & .927 & 0.945 & & & & & & & & & & & & \\
\hline 138 & 856 & 877 & 905 & & 0.936 & 0.950 & 900 & & & & & & & & & & \\
\hline 140 & 843 & 864 & 893 & .906 & 0.927 & 0.941 & & & & & & & & & & & \\
\hline 142 & 828 & 851 & 882 & .896 & 0.918 & 0.932 & & & & & & & & & & 95 & \\
\hline 144 & aN & 0.835 & 868 & 0.884 & 0.907 & 0.923 & & & & & & & & & & & \\
\hline 146 & & 0.820 & 853 & 0.869 & 0.893 & 0.910 & & & & & & & & & & & \\
\hline & & & 836 & .853 & 0.880 & & & & & & & & & & & & \\
\hline 150 & & & 819 & 027 & & & & & & & & & & & & & \\
\hline 152 & & & & & & & & & 100 & & & & & & & 991 & 0.991 \\
\hline 154 & $\mathrm{aN}$ & & & 800 & 0.834 & & & & & & & & & & & & \\
\hline 156 & $\mathrm{JaN}$ & 0.729 & 760 & 0.780 & 0.816 & 0.843 & & & & & & & & & & 987 & \\
\hline 158 & $\mathrm{NaN}$ & 0.707 & .735 & 0.757 & 0.796 & 0.824 & 866 & 0.966 & & & & & & & & 984 & \\
\hline 160 & 20 & & & 0.730 & & 0.803 & & & & & & & & & & & \\
\hline & 10 & $\mathrm{NaN}$ & 0.675 & & 0.746 & 0.781 & & & & & & & & & & & \\
\hline & & & & .009 & 0.721 & & & & & & & & & & & & \\
\hline 166 & aiv & div & aris & 0.632 & 0.691 & 0.734 & & & & & & & & & & .966 & \\
\hline 168 & $\mathrm{aN}$ & & & $\mathrm{IaN}$ & 0.661 & 0.708 & & & & & & & & & & 960 & \\
\hline 170 & $\mathrm{NaN}$ & $\mathrm{NaN}$ & $\mathrm{IaN}$ & $\mathrm{NaN}$ & & $\mathrm{N}$ & & & & & & & & & & & \\
\hline 172 & $\mathrm{NaN}$ & $\mathrm{NaN}$ & $\mathrm{NaN}$ & & & $\mathrm{NaN}$ & & & & & & & & & & & \\
\hline 174 & $\mathrm{NaN}$ & $\mathrm{NaN}$ & $\mathrm{NaN}$ & $\mathrm{NaN}$ & $\mathrm{NaN}$ & $\mathrm{N} / 2 \mathrm{~N}$ & 40 & & 0.929 & & & & & 0.918 & & .918 & \\
\hline 176 & INaiv & $\mathrm{NaN}$ & TIValv & $\mathrm{NaN}$ & $\mathrm{NaN}$ & INalv & & & & & & & & & & 1899 & \\
\hline 178 & $\mathrm{NaN}$ & $\mathrm{NaN}$ & $\mathrm{NaN}$ & $\mathrm{NaN}$ & $\mathrm{NaN}$ & TValv & Taiv & INalv & 0.946 & 0.904 & & 0.900 & 0.878 & 0.879 & & .882 & 0.884 \\
\hline ou & $\mathrm{NaN}$ & $\mathrm{NaN}$ & $\mathrm{NaN}$ & $\mathrm{NaN}$ & $\mathrm{VaN}$ & $\mathrm{NaN}$ & & & 0.972 & 0.886 & 0.932 & 0.898 & 0.858 & .861 & 0.863 & 0.864 & 0.867 \\
\hline
\end{tabular}


Table 3. Cont.

\begin{tabular}{|c|c|c|c|c|c|c|c|c|c|c|c|c|c|c|c|c|}
\hline \multicolumn{17}{|c|}{$\mathrm{rlcm}$} \\
\hline$\theta^{\circ}$ & 3 & 3.5 & 4 & 4.5 & 5 & 6 & 7 & 8 & 9 & 10 & 11 & 12 & 14 & 16. & 18 & 20 \\
\hline 90 & 1.000 & 1.000 & 1.000 & & 1.000 & & & & & 1.000 & & & & 1.000 & & \\
\hline & 1.000 & 1.000 & 000 & 000 & 1.001 & & & & & 1.001 & & & & .000 & 1.000 & \\
\hline 94 & 1.000 & 1.000 & 000 & 000 & 1.000 & 1.000 & 1.001 & 1.001 & 00 & 1.000 & 1.000 & 1.000 & & 000 & 1.000 & 01 \\
\hline 96 & 1.000 & 1.000 & 1.000 & 1.000 & 1.000 & 1.000 & 1.000 & 1.000 & 0.999 & 1.000 & 000 & 0.999 & 0.999 & 0.999 & 1.000 & 1.000 \\
\hline & 1.000 & 1.000 & 1.000 & 1.000 & 1.000 & 1.000 & 1.000 & 1.000 & 1.000 & 1.000 & 1.000 & 1.000 & 1000 & .000 & 1.000 & 1.000 \\
\hline (م) & 1.000 & 0909 & 1.000 & 1.000 & 1.000 & 1.000 & 1.000 & 1.000 & 0.999 & 1.000 & 1.000 & 1.000 & 0.999 & 1.000 & 0.999 & 100 \\
\hline 102 & 1.000 & 1.001 & 1.001 & 1.001 & & 1.001 & 1.001 & 1.001 & 1.000 & 1.001 & & & & .000 & 1.001 & \\
\hline 104 & 1.000 & 1.000 & 1.000 & 1.000 & 1.000 & 1.000 & 1.000 & 1.000 & 1.000 & 1.000 & 1.000 & & & 1.000 & 1.000 & 1.000 \\
\hline 106 & 1.000 & 1.000 & 1.001 & 1.001 & 1.000 & 1.001 & 1.001 & 1.001 & 1.001 & 1.001 & 1.001 & 1.001 & & .001 & .001 & 1.001 \\
\hline 108 & 1.000 & 1.000 & 1.000 & 1.000 & 1. & 1.000 & 000 & 00 & 00 & 1.000 & & & & & 00 & 000 \\
\hline 110 & 0.999 & 0.999 & 0.999 & 0.999 & 1.00 & 1.000 & 1.000 & 1.000 & 0.999 & 1.000 & 00 & bo & & 999 & 000 & 000 \\
\hline 112 & 0.999 & 0.999 & 000 & 000 & 0.999 & & 00 & 1.000 & & 000 & & & & & 000 & \\
\hline 114 & 1.000 & 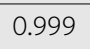 & 999 & & & & & 999 & & & & & & & & 999 \\
\hline 10 & & & & & & & & & & & & & & & & \\
\hline 18 & & 999 & 0.999 & 0.999 & & & 999 & 999 & 999 & 0.999 & & & & 999 & 999 & \\
\hline 20 & 999 & 0.999 & 0.999 & 0.999 & 999 & 999 & 00 & 999 & 0.999 & 0.999 & 999 & 999 & .999 & 999 & 999 & 0.999 \\
\hline 122 & 0.998 & .998 & 0.999 & 0.999 & .999 & 0.999 & .999 & .999 & 0.999 & 0.999 & 999 & .998 & 999 & 999 & 999 & 0.999 \\
\hline 124 & 0.999 & 0.999 & 0.999 & 0.999 & 0.999 & 0.999 & 0.999 & 0.999 & 0.999 & 0.999 & 999 & 0.999 & 0.999 & 999 & 999 & 0.999 \\
\hline 126 & 0.998 & 0.998 & 0.998 & 0.998 & 0.998 & 0.998 & 0.998 & 0.998 & 0.998 & 0.998 & 0.998 & 0.998 & 0.998 & 098 & 998 & 099 \\
\hline 128 & 0.998 & 0.998 & & & & & & & & & & & & & & \\
\hline 130 & & & & & & & & & & & & & & & & \\
\hline 32 & & & & & & & & & & & & & & & & \\
\hline 34 & & & & & & & & 997 & & 997 & & & & 97 & 997 & \\
\hline 36 & 0.997 & 0.997 & 0.997 & 0.997 & .997 & 0.997 & 997 & .997 & 0.996 & 0.997 & 997 & 0.997 & 0.997 & 997 & 0.997 & 0997 \\
\hline 38 & 0.996 & 0.996 & 0.996 & 0.996 & 0.996 & 0.996 & 996 & 0.996 & 0.996 & 0.996 & 0.996 & 0.996 & 0.996 & 0.996 & 0.996 & 009 \\
\hline 40 & 0.996 & 0.996 & 0.996 & 0.996 & 0.996 & 0.997 & 0.996 & 0.996 & 0996 & 0.996 & 0.996 & 0.996 & 0.996 & 0996 & 0.996 & 0006 \\
\hline 42 & 0.995 & 0.995 & 0.995 & 0.995 & 0.995 & 0.995 & 0.995 & 0.995 & 0.994 & 0.995 & 0.995 & R०० & 0.995 & 001 & 0.995 & 0 \\
\hline 144 & 0.995 & & 0.995 & 0.995 & 0.995 & & 0.995 & 0.995 & & & & & & & 0.995 & 0 \\
\hline 146 & 0.994 & 0.994 & .994 & 0.994 & 994 & 0.994 & 994 & 994 & & 994 & & & & & 994 & 94 \\
\hline 48 & 992 & 0.5 & & & & & & & & & & & & & 92 & 92 \\
\hline 50 & 0.991 & 0.991 & 991 & & & & & 991 & & 0.991 & & & & & 991 & 0.991 \\
\hline 52 & 0.991 & 0.991 & 0.991 & 0.991 & 0.991 & 0.991 & 0.991 & 0.991 & 0.991 & 0.992 & 0.992 & 0.991 & & 0.991 & 0.992 & 0007 \\
\hline 54 & 0.988 & 0.988 & 0.989 & 0.989 & 0080 & 0.989 & 989 & 0.989 & 0980 & 0.989 & 098 & 0000 & & & 0.989 & \\
\hline 56 & 0.987 & 0.987 & 0.987 & 0.987 & 097 & 0.987 & 987 & 0.987 & 0.987 & 0.987 & 0.987 & 0.901 & & & 0.988 & 0.900 \\
\hline 58 & 0.984 & 0.984 & 0.984 & & & & & 0.985 & & 0.985 & & & & & & \\
\hline 160 & 0.981 & 0.982 & 0.982 & 0.982 & 0.982 & & .983 & 0.982 & 0.983 & 0.983 & 0.983 & & & & 0.984 & 0.984 \\
\hline 162 & 0.977 & 0.977 & 977 & & 978 & & & & & 0.9 & & & & & 980 & 981 \\
\hline 164 & 0.973 & 0.974 & 0.974 & 0.974 & 0.974 & 0.975 & 975 & 0.975 & 0.976 & 0.976 & 976 & 977 & 0.978 & 978 & 0.979 & 0.979 \\
\hline 166 & 0.967 & 0.967 & 967 & 0.968 & 0.968 & & & 970 & & 0.971 & & & & & .973 & 0.974 \\
\hline 00 & 0.961 & & & & & & & & & & & & & & 969 & 0.970 \\
\hline 70 & 0.950 & 0.950 & 0.951 & 0.951 & 0.952 & & & 0.955 & & 0.957 & & & & & 0.963 & 0.963 \\
\hline 72 & 0.936 & 0.936 & 0.937 & 0.938 & 0.939 & 0.940 & .942 & 0.943 & 0.944 & 0.945 & 0.947 & & & & 0.953 & 0.955 \\
\hline 74 & 0.921 & 0.922 & 0.923 & 0.924 & 0.925 & 0.927 & 0.929 & 0.931 & 0.932 & 0.935 & 0.936 & & & 0.942 & 0.944 & 0.946 \\
\hline 76 & 0.902 & 0.904 & 0.906 & 0.907 & 0.909 & 0.911 & 0.914 & 0.916 & 0.918 & 0.920 & 0.923 & 0.925 & 0.928 & 0.932 & 0.935 & 0.935 \\
\hline 78 & 0.886 & 0.887 & 0.889 & 0.891 & 0.893 & 0.896 & & 0.902 & 0.904 & 0.907 & 0.908 & & & 0.919 & 0.922 & 0.914 \\
\hline & .869 & & & & 0.877 & 0.880 & & 0.889 & & 0.893 & 0.893 & 0.897 & 0.902 & 0.906 & 0.908 & כת \\
\hline
\end{tabular}


Table 4. Along away (cGy h-1 $\mathrm{U}^{-1}$ ) table calculated for the Flexisource Co-60 HDR source for QA purposes

\begin{tabular}{|c|c|c|c|c|c|c|c|c|c|c|c|c|c|c|}
\hline & & & & & & & & & & & & & & \\
\hline $\mathrm{z} / \mathrm{cm} 0.1$ & 0.2 & 0.3 & 0.4 & 0.5 & 0.6 & 0.7 & 0.8 & 0.9 & 1 & 2 & 4 & 6 & 8 & 10 \\
\hline $\begin{array}{ll}-10 & 0.00841\end{array}$ & 0.00842 & 0.00844 & 0.00846 & 0.00848 & 0.00850 & 0.00852 & 0.00855 & 0.00856 & 0.00859 & 0.00858 & 0.00778 & 0.00655 & 0.00531 & 0.00423 \\
\hline$-8 \quad 0.0136$ & 0.01365 & .01369 & 0.01373 & 0.01378 & 0.01381 & 0.01388 & 0.01392 & 0.01396 & 0.01398 & 0.01378 & 0.01178 & 0.00926 & & 0.00532 \\
\hline $\begin{array}{ll}-6 & 0.0249\end{array}$ & 0.0251 & 0.0252 & 0.0253 & 0.0255 & 0.0256 & 0.0257 & 0.0258 & 0.0258 & 0.0258 & 0.0245 & 0.0188 & 0.01329 & 0.00929 & 0.00659 \\
\hline $\begin{array}{ll}-4 & 0.0578\end{array}$ & 0.058 & 0.0589 & 0.059 & 0.060 & 0.060 & 0.060 & 0.060 & 0.060 & 0.059 & 0.051 & 0.0316 & 0.01890 & 0.01188 & 0.00789 \\
\hline $\begin{array}{ll}-2 & 0.241\end{array}$ & 0.246 & 0.249 & 0.249 & 0.247 & 0.242 & 0.236 & 0.229 & 0.221 & 0.213 & 0.1326 & 0.0517 & 0.0250 & 0.01418 & 0.00891 \\
\hline $\begin{array}{ll}-1 & 1.022\end{array}$ & 1.040 & 1.005 & 0.945 & 0.882 & 0.807 & 0.737 & 0.668 & 0.604 & 0.545 & 214 & 0.0611 & 0.0272 & 0.01489 & 0.00921 \\
\hline-0.91 .293 & 1.287 & 1.232 & 1.144 & 1.047 & 0.945 & 0.847 & 0.757 & 0.675 & 0.603 & 0.223 & 0.0619 & 0.0273 & 0.01494 & 0.00922 \\
\hline-0.81 .672 & 1.637 & 1.536 & 1.401 & 1.253 & 1.111 & 0.977 & 0.858 & 0.755 & 0.666 & 0.231 & 0.0625 & 0.0274 & 0.01498 & 0.00924 \\
\hline-0.72 .23 & 2.15 & 1.965 & 1.741 & 1.513 & 1.308 & 1.128 & 0.973 & 0.842 & & & 0.0631 & .0276 & 0.01502 & 0.00926 \\
\hline-0.63 .12 & 2.94 & 2.58 & 2.19 & 1.846 & 1.549 & 1.301 & 1.098 & 0.935 & 0.803 & 0.246 & 0.0636 & 0.0277 & 0.01506 & 0.00927 \\
\hline$-0.5 \mathrm{NaN}$ & 4.21 & 3.48 & 2.80 & 2.26 & 1.826 & 1.492 & 1.232 & 1.032 & 0.873 & 0.253 & 0.0641 & 0.0277 & 0.01508 & 0.00928 \\
\hline$-0.4 \mathrm{NaN}$ & 6.34 & 4.82 & 3.61 & 2.75 & 2.14 & 1.693 & 1.368 & 1.125 & 0.939 & 0.258 & 0.0645 & 0.0278 & 0.01510 & 0.00930 \\
\hline$-0.3 \mathrm{NaN}$ & 10.01 & 6.73 & 4.60 & 3.29 & 2.46 & 1.891 & 1.494 & 1.210 & 0.998 & 0.263 & 0.0647 & 0.0279 & 0.01512 & 0.00930 \\
\hline$-0.25 \mathrm{NaN}$ & 12.63 & 7.88 & 5.13 & 3.56 & 2.61 & 1.982 & 1.550 & 1.247 & 1.023 & & & 0.0279 & 0.01513 & 0.00931 \\
\hline$-0.2 \mathrm{NaN}$ & 15.69 & 9.06 & 5.64 & 3.82 & 2.74 & & & & & & & & 0.01514 & 0.00931 \\
\hline$-0.15 \mathrm{NaN}$ & 18.80 & 10.19 & 6.11 & 4.03 & 2.86 & 2.13 & 1.639 & 1.304 & 1.062 & 0.267 & 0.0650 & 0.0279 & 0.01514 & 0.00931 \\
\hline$-0.1 \mathrm{NaN}$ & 21.4 & 11.11 & 6.48 & 4.20 & 2.95 & 2.18 & 1.668 & 1.323 & 1.074 & 0.268 & 0.0651 & 0.0279 & 0.01514 & 0.00931 \\
\hline$-0.05 \mathrm{NaN}$ & 23.1 & 11.72 & 6.72 & 4.31 & 3.00 & 2.21 & 1.687 & 1.335 & 1.082 & 0.268 & 0.0651 & 0.0279 & 0.01514 & 0.00930 \\
\hline $\mathrm{NaN}$ & 23.7 & 11.93 & 6.80 & 4.35 & 3.02 & 2.22 & 1.692 & 1.339 & 1.085 & 0.269 & 0.0651 & 0.0279 & 0.01514 & 0.00930 \\
\hline $0.05 \mathrm{NaN}$ & 23.1 & 11.71 & 6.71 & 4.31 & 3.00 & 2.21 & 1.687 & 1.335 & 1.082 & 0.268 & 0.0651 & 0.0279 & 0.01514 & 0.00930 \\
\hline $0.1 \mathrm{NaN}$ & 21.3 & 11.09 & 6.47 & 4.20 & 2.94 & 2.18 & 1.668 & 1.323 & 1.074 & & 0.0651 & 0.0279 & 0.01514 & 0.00930 \\
\hline $0.15 \mathrm{NaN}$ & 18.67 & 10.16 & 6.10 & 4.03 & 2.85 & 2.13 & 1.638 & 1.304 & 1.062 & & & & 0.01513 & 0.00930 \\
\hline $0.2 \mathrm{NaN}$ & 15.52 & 9.04 & 5.64 & 3.81 & 2.74 & 2.06 & 1.598 & 1.278 & 1.044 & 0.266 & 0.0649 & 0.0279 & 0.01513 & 0.00930 \\
\hline $0.25 \mathrm{NaN}$ & 12.47 & 7.85 & 5.12 & 3.56 & 2.61 & 1.983 & 1.550 & 1.246 & 1.023 & 0.264 & 648 & .0279 & 0.01512 & 0.00930 \\
\hline 0.313 .49 & 9.89 & 6.70 & 4.59 & 3.29 & 2.46 & 1.894 & 1.494 & 1.210 & 0.997 & 0.263 & 0.0647 & 0.0279 & 0.01512 & 0.00930 \\
\hline $0.4 \quad 7.66$ & 6.31 & 4.80 & 3.60 & 2.74 & 2.14 & 1.697 & 1.367 & 1.125 & 0.939 & 0.258 & 0.0644 & 0.0278 & 0.01510 & 0.00929 \\
\hline $0.5 \quad 4.79$ & 4.20 & 3.47 & 2.80 & 2.25 & 1.825 & 1.491 & 1.232 & & 0.872 & & & & 0.01508 & 0.00928 \\
\hline $0.6 \quad 3.21$ & 2.94 & 2.57 & 2.19 & 1.846 & 1.546 & 1.299 & 1.098 & & 0.802 & & & 0.0277 & 0.01505 & 0.00927 \\
\hline $0.7 \quad 2.30$ & 2.16 & 1.964 & 1.739 & 1.515 & 1.309 & 1.128 & 0.972 & 0.842 & 0.732 & 0.239 & & 0.0275 & 0.01501 & 0.00926 \\
\hline $0.8 \quad 1.711$ & 1.645 & 1.535 & 1.397 & 1.250 & 1.107 & 0.975 & 0.858 & 0.755 & 0.665 & 0.231 & 0.0625 & .0274 & 0.01497 & 0.00924 \\
\hline 1.338 & 1.300 & 1.232 & 1.143 & 1.044 & 0.943 & 0.846 & 0.756 & 0.675 & 0.603 & 0.223 & 0.0618 & 0.0273 & 0.01493 & 0.00922 \\
\hline 1.075 & 1.051 & 1.009 & 0.950 & 0.881 & 0.809 & 0.736 & 0.667 & 0.603 & 0.545 & 0.214 & 0.0611 & 0.0272 & 0.01489 & 0.00920 \\
\hline 0.259 & 0.258 & 0.256 & 0.253 & 0.249 & 0.243 & 0.237 & 0.229 & 0.222 & 0.213 & 0.1326 & 0.0516 & 0.0250 & 0.01417 & 0.00891 \\
\hline 0.0622 & 0.0623 & 0.0623 & 0.0621 & 0.0619 & 0.0616 & 0.0613 & 0.0609 & 0.0604 & 0.0598 & 0.0512 & 0.0316 & 0.01890 & 0.01189 & 0.00789 \\
\hline 0.0267 & 0.0268 & 0.0268 & 0.0268 & 0.0267 & 0.0267 & 0.0266 & 0.0265 & 0.0264 & 0.0263 & 0.0246 & 0.01882 & 0.01330 & 0.00929 & 0.00659 \\
\hline 0.01454 & 0.01448 & 0.01456 & 0.01455 & 0.01451 & 0.01453 & 0.01449 & 0.01447 & 0.01441 & 0.01440 & 0.01388 & 0.01178 & 0.00926 & 0.00705 & 0.00532 \\
\hline 0.00892 & 20.00894 & 0.00893 & 0.00895 & 0.00894 & 0.00893 & 0.00894 & 0.00893 & 0.00891 & 0.00891 & 0.00870 & 0.00778 & .00655 & 0.00531 & 0.00423 \\
\hline
\end{tabular}

tion was used. Papagiannis et al. [23] reported alongaway dose rate tables and TG- 43 dose parameters. Selvam et al. [24] have reported a systematic error for $y=0.75 \mathrm{~cm}$ in the away-along table of Papagiannis et al. for the type 2 source model.

Ballester et al. [13] studied the GK60M21 ${ }^{60} \mathrm{Co}$ (Eckert \& Ziegler IBt-Bebig GmbH, Germany) using GEANT4 code to obtain the dose rate distribution around this source in an unbounded water phantom. Only the gamma part of the ${ }^{60} \mathrm{Co}$ spectrum was considered. The $\beta$ spectrum contribution to the dose was assumed to be insignificant. A cut-off energy of $10 \mathrm{keV}$ was used for both photons and electrons. They scored kerma and dose separately to account for the electronic disequilibrium near the source. For points located 

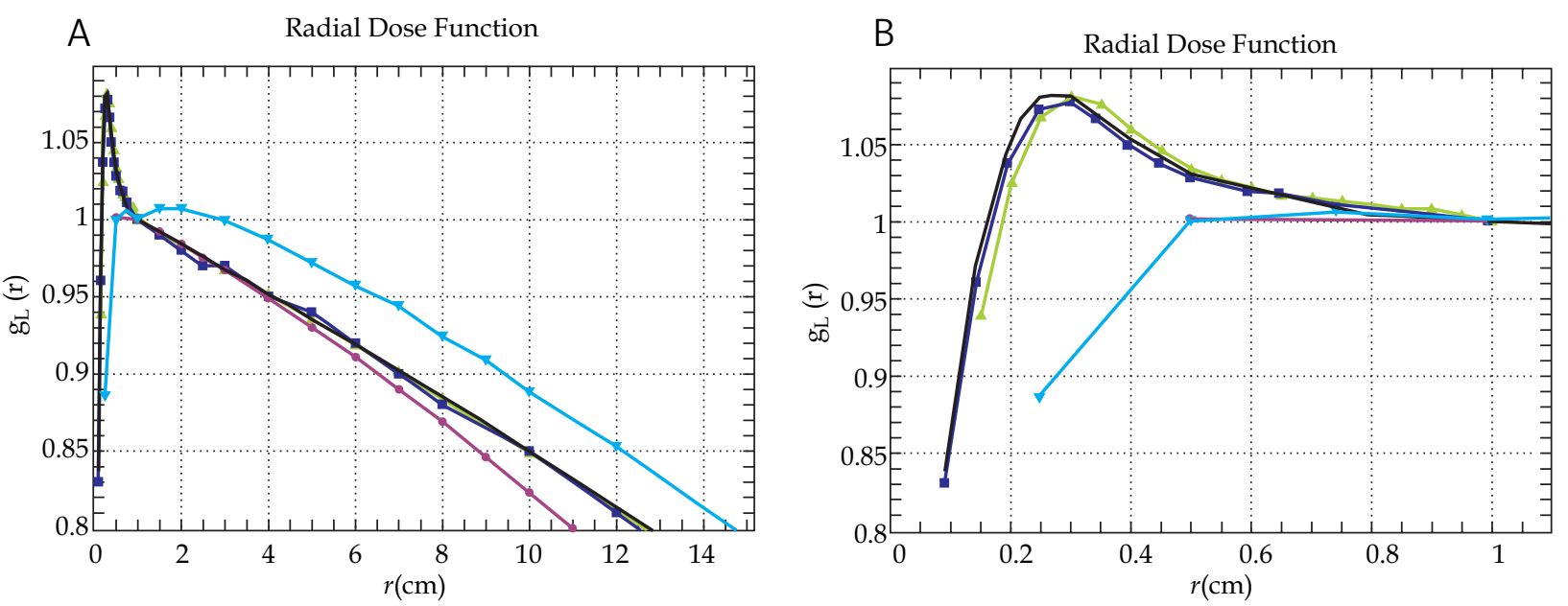

- Bebig Co60-A88 and GK60M21 Granero et al.
- Bebig Co60-A88 and GK60M21 Selvam et al.
- Type-2 Papagiannis et al.
- Flexisource Co-60
$\nabla$ GZP6 Tabrizi et al.

Fig. 2. A) Radial dose function of ${ }^{60}$ Co source models. B) Zoom-in at short distances from the source where the electronic disequilibrium is located

at distances of less than $1 \mathrm{~cm}$ from the source they scored dose, while for distances where electronic equilibrium was achieved they scored kerma. They derived TG-43U1 parameters and an away-along table. Selvam et al. [25] reproduced the Ballester et al. [13] study, but using the EGSnrc code. They derived only an away-along table. The comparison of away-along tables from both studies reveals consistency between both studies except at $y=0.25 \mathrm{~cm}$ and $z=-0.25, z=0$ and $z=0.25 \mathrm{~cm}$ were the Ballester et al. data had a typo.

Granero et al. [11] used GEANT4 MC code to obtain the dose rate distribution for the Co0.A86 ${ }^{60} \mathrm{Co}$ source model (Eckert \& Ziegler IBt-Bebig GmbH, Germany) in an unbounded water phantom. The same type of study that the Ballester et al. [13], one of the GK60M21 source model described in the previous paragraph was done for the Co0.A86 source model. Selvam et al. [25] also reproduced the Granero et al. [11] study, but using the EGSnrc code, obtaining only an away-along table. The comparison of away-along tables from both studies reveals that at $(y=0.25$ $\mathrm{cm}, z=-0.25 \mathrm{~cm}),(y=0.25 \mathrm{~cm}, z=0 \mathrm{~cm}),(y=0.25 \mathrm{~cm}$, $z=0.25 \mathrm{~cm}$ ), the Granero et al. [11] data are underestimated. This is the same typo as in Ballester et al. data [13] for the GK60M21 source model.

Tabrizi et al. [26] studied two different ${ }^{60} \mathrm{Co}$ linear braid type sources available for the GZP6 remote afterloader (Nuclear Power Institute of China). These sources are composed of one active core made of metallic ${ }^{60} \mathrm{Co}$ with $3.5 \mathrm{~mm}$ length and $1.5 \mathrm{~mm}$ diameter, encapsulated in $0.1 \mathrm{~mm}$ titanium. The active core is covered by a cylindrical stainless steel cover of $0.5 \mathrm{~mm}$ external diameter and steel balls arranged along. The authors used the MCNP4C Monte Carlo code to obtain the TG-43 dosimetric parameters together with along-away dose rate tables. Their radial dose function (see Fig. 2 in Tabrizi et al.) is inconsistent with other ${ }^{60}$ Co source data and is difficult to understand from a physical point of view.

Papagianis et al. [23] showed that $\Lambda / \mathrm{G}\left(r=1 \mathrm{~cm}, \theta=90^{\circ}\right)$ values for different ${ }^{60} \mathrm{Co}$ source models are expected to match each other providing the spatial dependence of the dose rate constant as removed. In Table 1, it can be observed that the Flexisource Co-60 HDR source fits into this scheme.

$g_{L}(r)$ for the Flexisource Co-60 HDR source is compared in Fig. 2A with corresponding data to GK60M21 [13] and Co0.A86 [11] sources from BEBIG, Ralstron HDR Type 2 [23] from Shimazdu and GZP6 sources [26]. Figure 2B illustrates similarities/differences for $r<1 \mathrm{~cm}$. The differences between Papagianis et al. data and those of the present study are due to the different phantom sizes used in the MC calculations. For the GZP6 source model data present an anomalous pattern.

Anisotropy function $F(r, \theta)$ is shown in Fig. 3 for $r \leq 1 \mathrm{~cm}$. At larger distances, $F(r, \theta)$ behave in a similar way as for $r=1 \mathrm{~cm}$.

\section{Conclusions}

A dosimetric study of the Nucletron Flexisource Co-60 HDR source for which no published dosimetric data existed was performed. TG- 43 parameters, dose rate constant, radial dose function and anisotropy function were provided together with a 2D along and away dose table. These datasets can be used either as an input for (or to validate) the TPS calculations essential for clinical practice. 


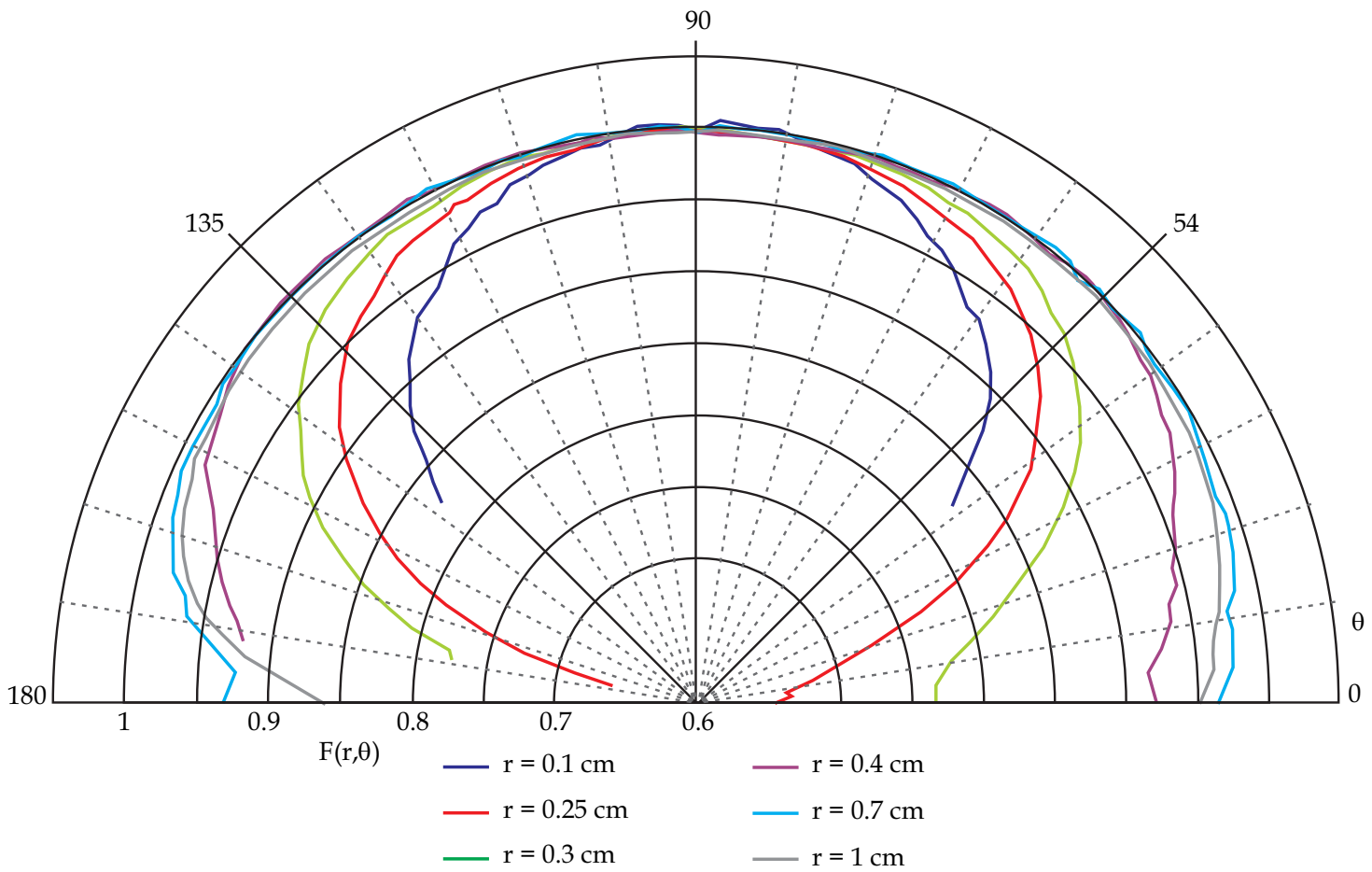

Fig. 3. Anisotropy function of the Flexisource Co-60 HDR source model for selected distances

\section{Acknowledgements}

This study was supported in part by Generalitat Valenciana (Project PROMETEO2008/114) FEDER, Ministerio de Ciencia e Innovación, Spain (Project No. FIS2010-17007) and by a research agreement with Nucletron B.V. Veenendaal, The Netherlands.

\section{References}

1. Li Z, Das RK, DeWerd LA et al.; American Association of Physicists in Medicine (AAPM); European Society for Therapeutic Radiology and Oncology (ESTRO). Dosimetric prerequisites for routine clinical use of photon emitting brachytherapy sources with average energy higher than $50 \mathrm{keV}$. Med Phys 2007; 34: 37-40.

2. Nath R, Anderson LL, Luxton G. et al. Dosimetry of interstitial brachytherapy sources: recommendations of the AAPM Radiation Therapy Committee Task Group No. 43. American Association of Physicists in Medicine. Med Phys 1995; 22 : 209-234.

3. Rivard MJ, Coursey BM, DeWerd LA et al. Update of AAPM Task Group No. 43 Report: A revised AAPM protocol for brachytherapy dose calculations. Med Phys 2004; 31: 633-674.

4. Ballester F, Granero D, Pérez-Calatayud J et al. Evaluation of high-energy brachytherapy source electronic disequilibrium and dose from emitted electrons. Med Phys 2009; 36: 4250-4256.

5. Baltas D, Karaiskos P, Papagiannis $P$ et al. Beta versus gamma dosimetry close to Ir-192 brachytherapy sources. Med Phys 2001; 28: 1875-1882

6. Wang R, XA Li. A Monte Carlo calculation of dosimetric parameters of ${ }^{90} \mathrm{Sr} /{ }^{90} \mathrm{Y}$ and ${ }^{192} \mathrm{Ir}$ SS sources for intravascular brachytherapy. Med Phys 2000; 27: 2528-2535.

7. Baltas D, Lymperopoulou G, Zamboglou N. On the use of HDR ${ }^{60} \mathrm{Co}$ source with the MammoSite radiation therapy system. Med Phys 2008; 35: 5263-5268.
8. Richter J, Baier K, Flentje M. Comparison of 60-cobalt and 192iridium sources in high dose rate afterloading brachytherapy. Strahlenther Onkol 2008; 184: 187-192.

9. Salvat F, Fernández-Varea JM, Sempau J. PENELOPE-2008: a code system for Monte Carlo simulation of electron and photon transport, 2008. Issy-les-Moulineaux, France.

10. GEANT4 Collaboration. GEANT4 - a simulation toolkit. Nucl Instrum Methohs Phys Res 2003; 506: 250-303.

11. Granero D, Perez-Calatayud J, Ballester F. Technical note: Dosimetric study of a new Co- 60 source used in brachytherapy. Med Phys 2007; 34: 3485-3488.

12. Granero D, Perez-Calatayud J, Pujades-Claumarchirant MC et al. Equivalent phantom sizes and shapes for brachytherapy dosimetric studies of ${ }^{192}$ Ir and ${ }^{137}$ Cs. Med Phys 2008; 35: 4872-4877.

13. Ballester F, Granero D, Pérez-Calatayud J et al. Monte Carlo dosimetric study of the BEBIG Co-60 HDR source. Phys Med Biol 2005; 50: N309-N316.

14. Granero D, Vijande J, Ballester F et al. Dosimetry revisited for the HDR ${ }^{192}$ Ir brachytherapy source model mHDR-v2. Med Phys 2011; 38: 487-494.

15. Meigooni AS, Wright C, Koona RA et al. TG-43 U1 based dosimetric characterization of model 67-6520 Cs-137 brachytherapy source. Med Phys 2009; 36: 4711-4719.

16. Cullen DE, Hubbell JH, Kissel L. EPDL97: the Evaluated Photon Data Library, '97 Version, 1997, University of California, Lawrence Livermore National Laboratory: Livermore, CA.

17. Cullen DE, Perkins ST, Seltzer SM. Tables and graphs of electron-interaction cross-sections from $10 \mathrm{eV}$ to $100 \mathrm{GeV}$ derived from the LLNL Evaluated Electron Data Library (EEDL) Z=1100, 2001, Lawrence Livermore National Laboratory.

18. NUDAT 2.5, National Nuclear Data Center, 2010, Brookhaven National Laboratory.

19. Rivard MJ, Granero D, Perez-Calatayud J et al. Influence of photon energy spectra from brachytherapy sources on Monte 
Carlo simulations of kerma and dose rates in water and air. Med Phys 2010; 37: 869-876.

20. Badal A, Sempau J. A package of Linux scripts for the parallelization of Monte Carlo simulations. Comput Phys Commun 2006; 175: 10.

21. Williamson JF. Monte Carlo evaluation of kerma at a point for photon transport problems. Med Phys 1987; 14: 567-576.

22. Perez-Calatayud J, Granero D, Ballester F. Phantom size in brachytherapy source dosimetric studies. Med Phys 2004; 31 : 2075-2081.

23. Papagiannis P, Angelopoulos A, Pantelis E et al. Monte Carlo dosimetry of ${ }^{60} \mathrm{Co}$ HDR brachytherapy sources. Med Phys 2003; 30: 712-721.

24. Selvam TP, Sahoo S, Vishwakarma RS. Comment on "Monte Carlo dosimetry of ${ }^{60}$ Co HDR brachytherapy sources" [Med Phys 30, 712-721 (2003)]. Med Phys 2010; 37: 5146-5147.

25. Selvam TP, Bhola S. Technical note: EGSnrc-based dosimetric study of the BEBIG ${ }^{60}$ Co HDR brachytherapy sources. Med Phys 2010; 37: 1365-1370.

26. Hariri Tabrizi S, Kamali Asl A, Azma Z. Monte Carlo derivation of AAPM TG-43 dosimetric parameters for GZP6 Co-60 HDR sources. Phys Med 2012; 28: 153-160. 